\title{
Article \\ A Comparative Analysis of Wi-Fi Offloading and Cooperation in Small-Cell Network
}

\author{
Ayesha Ayub ${ }^{1}$, Sobia Jangsher ${ }^{1}$, M. Majid Butt ${ }^{2}$, Abdur Rahman Maud ${ }^{1}$ and Farrukh A. Bhatti ${ }^{1, *}$ (i) \\ 1 WiSP Lab, Electrical Engineering Department, Institute of Space Technology, Islamabad 44000, Pakistan; \\ ayeshamirza5252@gmail.com (A.A.); sobia.jangsher@ist.edu.pk (S.J.); a.rahman@mail.ist.edu.pk (A.R.M.) \\ 2 Trinity College Dublin, D02 PN40 Dublin, Ireland; majid.butt@tcd.ie \\ * Correspondence: farrukh.aziz@ist.edu.pk
}

Citation: Ayub, A.; Jangsher, S.; Butt, M.M.; Maud, A.R.; Bhatti, F.A. A Comparative Analysis of Wi-Fi Offloading and Cooperation in Small-Cell Network. Electronics 2021, 10, 1493. https://doi.org/10.3390/ electronics10121493

Academic Editor: Khaled Elleithy

Received: 23 May 2021

Accepted: 15 June 2021

Published: 21 June 2021

Publisher's Note: MDPI stays neutral with regard to jurisdictional claims in published maps and institutional affiliations.

Copyright: (c) 2021 by the authors. Licensee MDPI, Basel, Switzerland. This article is an open access article distributed under the terms and conditions of the Creative Commons Attribution (CC BY) license (https:// creativecommons.org/licenses/by/ $4.0 /)$.

\begin{abstract}
Small cells deliver cost-effective capacity and coverage enhancement in a cellular network. In this work, we present the interplay of two technologies, namely Wi-Fi offloading and small-cell cooperation that help in achieving this goal. Both these technologies are also being considered for 5G and B5G (Beyond 5G). We simultaneously consider Wi-Fi offloading and small-cell cooperation to maximize average user throughput in the small-cell network. We propose two heuristic methods, namely Sequential Cooperative Rate Enhancement (SCRE) and Sequential Offloading Rate Enhancement (SORE) to demonstrate cooperation and Wi-Fi offloading, respectively. SCRE is based on cooperative communication in which a user data rate requirement is satisfied through association with multiple small-cell base stations (SBSs). However, SORE is based on Wi-Fi offloading, in which users are offloaded to the nearest Wi-Fi Access Point and use its leftover capacity when they are unable to satisfy their rate constraint from a single SBS. Moreover, we propose an algorithm to switch between the two schemes (cooperation and Wi-Fi offloading) to ensure maximum average user throughput in the network. This is called the Switching between Cooperation and Offloading (SCO) algorithm and it switches depending upon the network conditions. We analyze these algorithms under varying requirements of rate threshold, number of resource blocks and user density in the network. The results indicate that SCRE is more beneficial for a sparse network where it also delivers relatively higher average data rates to cell-edge users. On the other hand, SORE is more advantageous in a dense network provided sufficient leftover Wi-Fi capacity is available and more users are present in the Wi-Fi coverage area.
\end{abstract}

Keywords: cooperative small-cell network; Wi-Fi offloading; LTE-U; heterogenous network

\section{Introduction}

Mobile network usage is increasing at a rapid rate of more than $40 \%$ compound annual growth rate. This is mainly due to the growing usage of video, online gaming, live streaming and multimedia applications etc., while the virtual reality and augmented reality applications are looming on the horizon. COVID-19 has further hyped up this demand as online video meetings and lecturing has increased significantly. As the use of social media has become widely popular, over-the-top players, such as Facebook and WhatsApp, have also increased their dominance in core communication services such as messaging and voice communication. This has resulted in significant decline in communication services revenues for the mobile network operators (MNOs). Therefore, it has become even more important for the MNOs to keep their CAPEX and OPEX low and rely on those technologies that offer wireless capacity enhancement with relatively lower investments. In this respect, Wi-Fi offloading and base station cooperation (or coordination) are very effective strategies. Wi-Fi offloading allows the MNOs to leverage the unlicensed spectrum, whereas cooperation helps in efficient use of licensed resources through coordination between the base stations. 
Several technologies have emerged recently that involve cellular data traffic offloading to Wi-Fi; however, LTE-Unlicensed (LTE-U) and License-Assisted Access (LAA) are the most notable [1,2]. LTE-U performs adaptive duty cycling with Carrier Sense Adaptive Transmission (CSAT), and adapts according to the Wi-Fi traffic load; however, it does not perform Listen Before Talk (LBT) [3]. LAA on the other hand does perform LBT, hence, it is considered to be better in maintaining fairness with Wi-Fi devices. Both LTE-U and LAA operate in 5GHz band and have been standardized in 3GPP Rel-12 and Rel-14 respectively [4]. Several studies have tried to analyze the fairness between LTE-U/LAA and Wi-Fi. The FCC report on the co-existence of LAA and Wi-Fi indicated that LAA could adversely affect throughput of Wi-Fi in an unfair manner [5]. In [6], the fairness between $\mathrm{Wi}-\mathrm{Fi}$ and LTE-U/LAA was investigated and it was concluded that when configured optimally, LTE-U and LAA could provide the same level of fairness as other Wi-Fi devices.

Coordinated Multipoint (CoMP) helps in improving coverage and cell-edge data rates and minimizes intercell interference through coordination between the base stations [7]. CoMP is also sometimes referred to as cooperative communication. While using CoMP, various transmission or reception points, such as SBS, relaying nodes or remote radio heads (RRH), can be coordinated to provide efficient service to the user equipment (UE). For example, data can be simultaneously transmitted in the same resource blocks from more than one SBS to a single UE, or data can be received by a UE from one SBS in one subframe and from another SBS in the next subframe [8]. Thus, CoMP enables the optimum paths for uplink and downlink traffic. CoMP has two main approaches; the first is based on interference mitigation and this includes coordinated scheduling (CS) and coordinated beamforming. The second approach is based on fusion, in which the base stations simultaneously transmit to an intended UE, and their streams are fused using joint transmission (JT) or joint processing (JP). CoMP implementation requires additional overhead communication due to exchange of channel state information (CSI), scheduling complexity and additional backhaul limitations. JT/JP typically requires additional transmit power to deliver capacity gains, especially for cell-edge UEs $[9,10]$.

In this paper, we present the comparison of Wi-Fi offloading and cooperative communication in a small-cell network. The proposed techniques can be adopted to enhance the QoS of the network and they can be implemented using network function virtualization (NFV) and software-defined networking (SDN) on top of the standard infrastructure. Due to the QoS enhancement of these strategies, we base our analysis of the two schemes as a QoS maximization problem. We consider sum data rate as our QoS measure and formulate the two problems individually. For Wi-Fi offloading, the data rate maximization relies mainly on the Wi-Fi leftover capacity, whereas, for cooperative communication, the number of associations of a user with small-cell (SC) base stations is the key factor. After analyzing each of the scenario, switching criteria is set for switching scheme.

The rest of the paper is structured as follows: Section 2 presents related research work. Section 3 describes the transmission models for both cooperation and Wi-Fi Offloading. Section 4 presents our proposed heuristics i.e., SCRE and SORE for the case of cooperation and offloading. Results and performance analysis is discussed in Section 5. Section 6 provides the future directions and conclusion.

\section{Related Work}

In this section, we present recent studies that have explored rate maximization in cooperative communication and Wi-Fi offloading. The related work for this paper can be categorized in two areas, i.e., cooperative communication and Wi-Fi offloading and we discuss each of them separately.

\subsection{Cooperative Small Cells}

BS cooperation is being considered for $5 \mathrm{G}$ and beyond 5G (B5G) for efficient coverage and high throughput. For B5G, cell-free system is being proposed where a group of base stations cooperatively serve the users without creating autonomous cells while intelligently 
identifying the user's communication environments [11-14]. Base station cooperation technologies are also critical for providing reliable cell-edge service in the millimeter wave bands, even for high mobility scenarios. In [15], SBS cooperation is implemented with digital beamforming in millimeter wave band.

For 5G/B5G systems data are cooperatively transmitted by multiple License-assisted access (LAA) SBSs through the unlicensed spectrum [16]. To improve the access to unlicensed spectrum, a coordinated access to the unlicensed spectrum is proposed using learning-assisted clustering of densely deployed SBSs. The CSI of LAA users in unlicensed band is fed back to the control plane of the (5G/B5G) system, and data are cooperatively transmitted by multiple LAA SBSs. Han et al. [17] analyzed small-cell base station (SBS) cooperation for software-defined hetnets using different metrics including connectivity probability, load balancing and energy efficiency. Chen et al. [18] proposed a joint transmission coordinated multipoint (JT-CoMP) technique that addresses the time synchronization issue. As described earlier, CoMP is a form of SBS cooperation, and it is being actively explored for implementation in 5G/B5G systems. Li et al. [12] analyze coordinated beamforming/scheduling scheme for massive MIMO small-cell network and their analysis shows $2-4.5$ times higher average sum-rate compared to non-cooperative massive MIMO network. This signifies SBS cooperation for future systems that are likely to have massive MIMO-based SBS.

In [19], by controlling user association, resource and power allocation, the authors aim to maximize the sum rate while also considering intercell interference coordination. In [20], the authors solve non-convex sum-rate maximization problem while considering limited backhaul capacity of small-cell BSs. In [20], the authors solve maximization problem related to the proportional fairness of cooperative multi cell network. The author of [21] optimizes association of user for load balancing by using CoMP techniques in cellular networks. A joint user association with JP-CoMP scheme for proportional fairness maximization among users of a network within a small-cell cluster using hybrid self-organizing network $(\mathrm{SON})$ is proposed in [22]. It is argued that the proposed scheme is effective (in terms of reducing the intercell interference) in handling the load balancing of the network. In [23], a mathematical framework for a user-centric small-cell network is proposed. The authors argue that Poisson Point Process is not a rigorous model when cooperation of small cells is involved. They also compute an optimal number of small cells for each user that maximizes the energy efficiency.

The cooperative small-cell concept has also been explored for enhancing caching in small-cell networks. The authors in [24] propose a high-performance architecture for fast content delivery using femto caching along with femto BS cooperation. If a user requests certain video file that is cached, then the femto BSs with the file cached will cooperate to transmit the information to the user. An energy efficient caching for a cooperative small cell is proposed in [25]. A file placement strategy is investigated such that the cooperation can enhance the overall energy efficiency of the network.

\subsection{Wi-Fi Offloading in Small Cells}

Substantial research has been performed on Wi-Fi offloading due to the benefits associated with offloading mobile network data to Wi-Fi that mainly include BS power saving, easing of network congestion and quality-of-service improvement for the users, especially in dense areas. Work in this area started more than a decade ago; however, due to its overwhelming advantages and network upgrades, research in this area still continues and Wi-Fi offloading is also being considered for 5G and B5G. Yi et al. [26] proposed a bid-based heterogeneous resource allocation framework that enables MNOs to efficiently use both cellular and operator-owned Wi-Fi resources simultaneously. An auction-based mechanism is used to achieve dynamic Wi-Fi offloading with awareness of user valuations. The operator-domain offloading helps avoid selfish user behavior and ensures near-optimal profit and social utility. Wehnao et al. [27] proposed a scheme for joint Wi-Fi and cellular 
offloading to optimally reduce the energy consumption and latency of mobile terminals in task processing.

Han proposed a Wi-Fi offloading algorithm based on traffic congestion awareness in [28]. The algorithm assumes user traffic information based on usage pattern (e.g., video streaming or emails etc.), i.e., user priority level. Users with high priority level are offloaded to $\mathrm{Wi}$-Fi on priority. However, it does not describe the overhead signaling involved in updating the priority level information which likely varies over time. Zhou et al. [29] proposed an incentive mechanism to encourage Wi-Fi AP owners to participate in data offloading. Their scheme (Delay-constraint and Reverse Auction-based Incentive Mechanism) solves an optimization problem to maximize the revenue of the $\mathrm{MNO}$ while jointly considering the delay constraint for various user applications. A Vickrey-Clarke-Groves scheme-based payment rule is also proposed to guarantee the individual rationality and truthfulness properties of users while considering different traffic load scenarios. Feng et al. [30] proposed an optimal pricing scheme to maximize the user satisfaction through Wi-Fi offloading and minimize the monetary losses to MNO incurred due to users offloading to Wi-Fi.

Previous works have individually considered cooperative SC network or Wi-Fi offloading for rate maximization. However, no work has considered the conditions in which either of the techniques is more useful compared to the other. In this work, we provide a comparison of the two schemes and discuss the scenarios where one is beneficial over the other.

\subsection{Contribution}

SBS cooperation is a vital technology being considered for 5G and B5G in the context of cell-free communication. Wi-Fi offloading is also being actively explored for 5G and B5G to complement cellular network throughput and coverage. Our study simultaneously considers both technologies for average data rate enhancement while considering different network conditions, which has not been considered in the previous studies. The specific contributions of this paper are as follows.

- We formulate a resource allocation problem for Wi-Fi offloading and SBS cooperation for co-located Wi-Fi access points (APs) and SBSs. In the case of Wi-Fi offloading, allocation of leftover capacity from the Wi-Fi network to the LTE UEs is considered to be an optimization variable, whereas for SBS cooperation the association with multiple SBSs is considered along with OFDM resource block allocation and power allocation problem.

- We propose heuristic algorithms based on sequential allocation of resources for both offloading and cooperation scenarios. The heuristic algorithms are called sequential cooperative rate enhancement (SCRE) and sequential offloading rate enhancement (SORE) for small-cell cooperation and Wi-Fi offloading, respectively.

- We present a comparative analysis between SCRE and SORE for maximizing the average user data rate depending on the network conditions. We conclude from the results that cooperation (SCRE) is more beneficial in less dense network where several users are at cell edge and the average data rate requirement is high. However, Wi-Fi offloading (SORE) is more advantageous when the density of cellular UEs is higher and more UEs are present within Wi-Fi range and there is also sufficient Wi-Fi leftover capacity available.

\section{System Model}

In this section, we present the system model for both cooperative communication and Wi-Fi offloading. An OFDMA-based downlink within a sector of a two-tier macro cell (MC)SC network is considered with N SCs and M UEs. The SCs consider in the MC network are placed adjacent to each other and $K$ unique OFDMA resource blocks (smallest unit of an OFDMA system) are assigned to each of them. We denote the small-cell BSs, UEs and RBs set as $\mathcal{I}=\{1,2, \ldots, M\}, \mathcal{J}=\{1,2, \ldots, N\}$ and $\mathcal{K}=\{1,2, \ldots, K\}$, respectively. A time interval $T$ is considered for which the resources are allocated in both the strategies while 
assuming quasi-static channel conditions. As already mentioned, this paper compares cooperative communication with Wi-Fi offloading. These two scenarios are shown in Figure 1a,b, respectively. Table 1 describes the notations used throughout the paper.

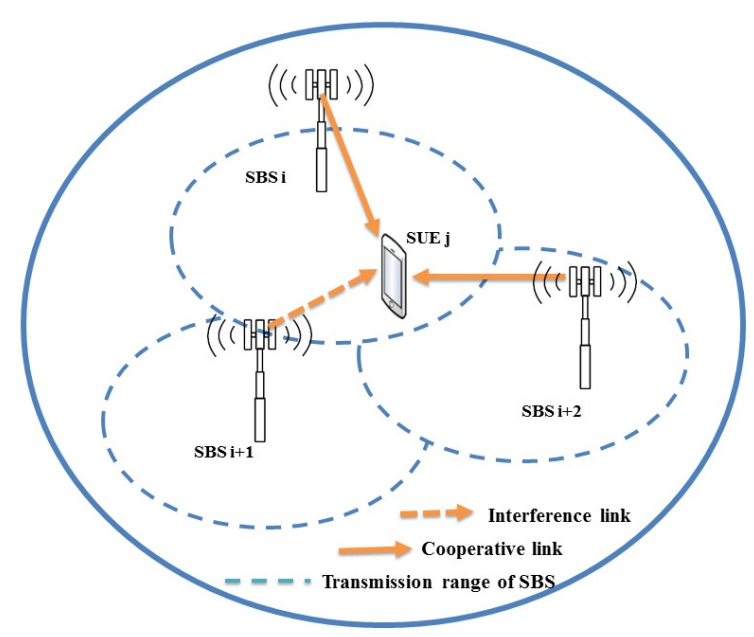

(a) Cooperation Communication

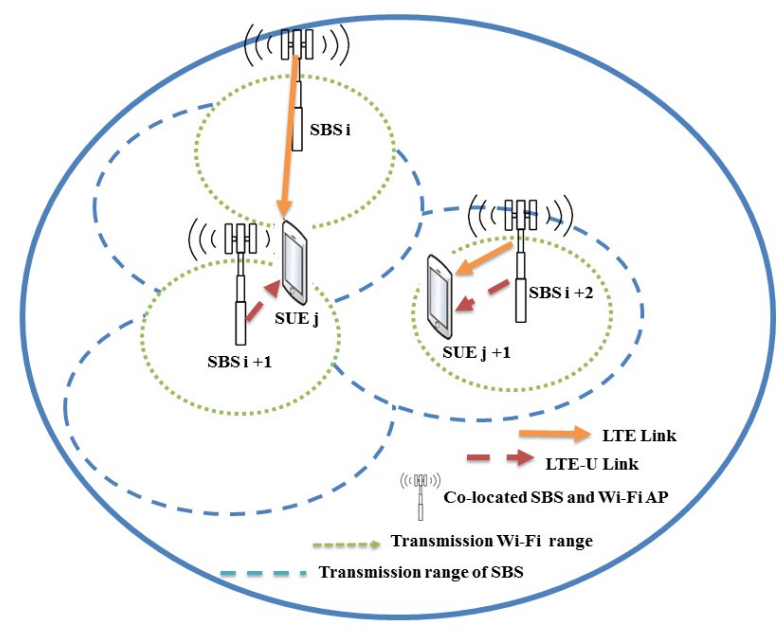

(b) Wi-Fi Offloading

Figure 1. Transmission scenarios for data rate enhancement in SC network.

Table 1. Explanation of Notations.

\begin{tabular}{ll}
\hline Notations & Explanation \\
\hline$I$ & Set of SCs in a MC \\
$J$ & Set of UEs in SCs \\
$P_{i j}^{k}$ & Power in downlink from a SBS $i$ to a UE $j$ on a RB $k$ \\
$G_{i j}$ & Gain directed from a SBS $i$ to a UE $j$ \\
$K$ & Vector with RBs \\
$x_{i j}^{k}$ & Association of a UE $j$ to a SBS $i$ on a RB $k$ \\
$y_{i j}^{k}$ & Association of a UE $j$ to a LTE SBS $i$ on a RB $k$ \\
$z_{i j}$ & Association of a UE $j$ to a LTE-U SBS $i$ \\
$\sigma^{2}$ & Noise power \\
$\gamma_{i j}^{k}$ & Signal-to-interference plus noise of a UE $j$ using a RB $k$ of a SBS $j$ \\
$R_{i j}^{k}$ & Achievable data rate to a UE $j$ using a RB $k$ of a SBS $i$ \\
$R_{j}^{c}$ & Achievable data rate to a UE $j$ using Cooperation \\
$R_{j}^{o}$ & Achievable data rate to a UE $j$ using Wi-Fi offloading \\
$R_{A P_{i}}$ & Total rate of a LTE-U SBS $i$ \\
$R_{j}^{t h}$ & Threshold Data rate for a UE $j$ \\
$M_{j}$ & Threshold number of SBSs can serve a UE $j$ \\
$N_{i}$ & Threshold number for UEs associated with a SBS $i$ \\
\hline
\end{tabular}

\subsection{Transmission Model for Cooperative Small-Cell Network}

Figure 1a shows the transmission scenario for cooperative SC network. In this network, each user can be served by more than one small-cell BSs to enhance its quality-of-service (QoS). As shown in Figure 1a, three SCs bounded with dashed lines are present in a MC. User $j$ is served cooperatively by SBS $i$ and $i+2$ through multiple links as illustrated with solid lines. However, SBS $i+1$ creates interference for UE $j$. In this paper, we consider achievable data rate as a measure of QoS for a user. In the case of cooperative communication, a joint problem of user association with multiple SCs, RB allocation and 
power allocation is studied. Here central BS (macro BS in this case) knows all the channel conditions. The optimization/control variable considered are as follows:

$$
x_{i j}^{k}= \begin{cases}1, & \text { if a UE } j \text { is associated with a SBS } i \text { on a RB } k, \\ 0, & \text { otherwise }\end{cases}
$$

and power allocation variable $P_{i j}^{k}$ which can be allocated a value in range of $0 \leq P_{i j}^{k} \leq P_{i}^{\max }$. Dimensions of association matrix and power allocation are $\mathbf{X}[M \times N \times K]$ and $\mathbf{P}[M \times N \times K]$ respectively. The signal-to-interference plus noise (SINR) ratio of a UE $j$ associated with a $\mathrm{SBS} i$ on a RB $k$ is given as:

$$
\gamma_{i j}^{k}=\frac{P_{i j}^{k} G_{i j}}{\sigma^{2}+\sum_{i^{\prime} \in I, i^{\prime} \neq i} P_{i^{\prime} j}^{k} G_{i^{\prime} j}},
$$

where $P_{i j}^{k}$ is the power transmitted from a SBS $i$ to a UE $j$ on a RB $k, G_{i j}$ is the channel gain experienced by a UE $j$ from a SBS $i, \sum_{i^{\prime} \in I, i^{\prime} \neq i} P_{i j}^{k} G_{i j}$ is the interference power received from all the remaining cells in set $I$ and $\sigma^{2}$ is the additive white Gaussian Noise. A physical interference model is considered to be presented in [31]. According to this model, two users cannot share the same resources if the SINR between the two is above a certain threshold. We use normalized data rates expressed as,

$$
R_{i j}^{k}=\log _{2}\left(1+\gamma_{i j}^{k}\right) .
$$

Equation (3) represents the achievable data rate of a UE $j$ from a SBS $i$ on a RB $k$. The achievable data rate of a UE over all SBS and all RBs using cooperative communication is represented as follows:

$$
R_{j}^{c}=\sum_{i \in I} \sum_{k \in K} x_{i j}^{k} R_{i j}^{k}
$$

where $x_{i j}^{k}$ is an association and RB allocation indicator and which is equal to 1 when a UE $j$ is associated with a SBS $i$ on a RB $k$ and is 0 otherwise. $R_{j}^{c}$ represents the achievable rate to a UE $j$ using cooperative communication.

\subsection{Transmission Model for Wi-Fi Offloading in Small Cells}

Figure $1 \mathrm{~b}$ shows the transmission model for the Wi-Fi offloading strategy in the SC network. Here the SBS and Wi-Fi AP are co-located with licensed band for cellular UEs and unlicensed band for Wi-Fi only (non-cellular) UEs. In this model, we assume that the Wi-Fi APs are either user deployed, or they might be deployed by another entity such as city council. Thus, the Wi-Fi operation does not incur any cost to the cellular operator. For convenient distinction, we refer to the Wi-Fi only UEs as Wi-Fi devices. Cellular UEs can also be offloaded to Wi-Fi (LTE-U). As shown in Figure 1b, three SC bounded with dashed lines are present in a MC. Each Wi-Fi BS has its own coverage region shown with a dotted line. UE $j$ connects with a single SBS $i$ using LTE downlink shown with a solid line to meet its data rate demand. In the case that it is unable to meet rate threshold then it is offloaded to LTE-U link of SBS $i+2$ because it lies within the Wi-Fi range of that SBS. Similarly, UE $j+1$ connects with a single SBS $i+1$ using LTE downlink and in addition it can be offloaded to the Wi-Fi AP co-located with the SBS $i+1$ to satisfy its data rate requirement. This Wi-Fi AP is also referred to as LTE-U SBS. In the SC network with Wi-Fi offloading, a joint problem involving user association with SBS, RB allocation, power allocation and leftover capacity is analyzed. The variables that indicate the UE's association with LTE SBS and LTE-U SBS are

$$
y_{i j}^{k}= \begin{cases}1, & \text { if a UE } j \text { is associated with LTE SBS } i^{\text {th }} \text { on a RB } k, \\ 0, & \text { otherwise }\end{cases}
$$


and

$$
z_{i j}= \begin{cases}1, & \text { if a UE } j \text { is associated with LTE-U of SBS } i, \\ 0, & \text { otherwise. }\end{cases}
$$

The power allocation variable $P_{i j}^{k}$ can be allocated a value between 0 and $P_{i}^{\max }$. Dimensions of association matrices are $\mathbf{Y}[M \times N \times K], \mathbf{Z}[M \times N]$ and the dimensions of the power allocation matrix is $\mathbf{P}[M \times N \times K]$. Data rate $R_{i j}^{U}$ that can be achieved by a UE $j$ from a Wi-Fi $\mathrm{AP} i$ is one of our controlling variables. We assume that the leftover capacity of each Wi-Fi $\mathrm{AP}$ is known to MBS. The leftover capacity of an AP is defined as $L_{i}=R_{A P_{i}}-\sum_{w \in W} r_{i}^{w}$ as in [32]. Here $R_{A P_{i}}$ is the total capacity of a Wi-Fi AP $i$ and $r_{i}^{w}$ is the data rate achieved by a Wi-Fi device $w$. Hence, the leftover capacity is equal to the rate that can be achieved by a UE (on LTE-U link) after all the Wi-Fi devices have satisfied their required data rates. The leftover capacity typically depends on the number of Wi-Fi devices and their average data rate requirement. The leftover capacity of each user is expressed as $R_{j}^{U}$. As the number of Wi-Fi devices increase, the leftover capacity reduces and vice-versa. SINR of a cellular UE using licensed band can be represented as $\gamma_{i j}^{L T E, k}$ as:

$$
\gamma_{i j}^{L T E, k}=\frac{P_{i j}^{k} G_{i j}}{\sigma^{2}+\sum_{i^{\prime} \in I_{j}, i^{\prime} \neq i} P_{i^{\prime} j}^{k} G_{i^{\prime} j}},
$$

where $P_{i j}^{k}$ is the power transmitted from a SBS $i$ to a UE $j$ on a RB $k$ and $G_{i j}$ is the channel gain experienced by a UE $j$ from a SBS $i$. We use normalized rates in this model. Moreover, we also use Rayleigh fading channels in our model. The required power for a UE $j$ is a product of power received by using a RB $k$ of a SBS $i$ and gain $G_{i j}$ from a SBS $i$ to a UE $j . \sigma^{2}$ is the noise power and $\sum_{i^{\prime} \in I, i^{\prime} \neq i} P_{i j}^{k} G_{i j}$ is interference power received from all the remaining SBSs in set $I$. The achievable data rate of a UE $j$ using a RB $k$ of LTE band of a SBS $i$ is represented as:

$$
R_{i j}^{L T E, k}=\log _{2}\left(1+\gamma_{i j}^{L T E, k}\right),
$$

and the data rate achieved over all SBSs over LTE band using all RBs is represented as:

$$
R_{j}^{L T E}=\sum_{i \in I} \sum_{k \in K} y_{i j}^{k} R_{i j}^{L T E, k},
$$

where $y_{i j}^{k}$ is an association indicator of a UE $j$ of a SBS $i$ on LTE band $k$ as in (5). The achievable data rate by a UE $j$ on unlicensed band is represented as:

$$
R_{j}^{U}=\sum_{i \in I} z_{i j} R_{i j}^{U}
$$

where $z_{i j}^{k}$ is an association indicator of a UE $j$ on LTE-U band of a SBS $i$ as defined in (6). The achievable data rate by a UE $j$ while using both bands LTE and LTE-U of a SBS is represented as:

$$
R_{j}^{o}=R_{j}^{L T E}+R_{j}^{U} .
$$

The overall system performance is depicted by finding the overall data rate achieved by all the cellular UEs in Wi-Fi offloading SC network as:

$$
R^{o}=\sum_{j \in J} R_{j}^{o},
$$

where $R_{j}^{o}$ represents the rate achieved by a UE $j$ while simultaneously using the LTE band and Wi-Fi offloading. Thus, the cellular UE initially tries to satisfy its rate requirement through LTE link, failing to do so it offloads to Wi-Fi AP to leverage its unused capacity. 


\section{Comparison between Cooperation and Wi-Fi Offloading in Small-Cell Network}

In this section, we present the problem formulation of cooperation and Wi-Fi offloading strategies separately and propose solutions to solve the formulated problems. Cooperation and Wi-Fi offloading are sophisticated algorithms of the two-tiered network to further enhance the data rate experienced by UEs in the network. We consider these two data rate enhancement strategies as these can be added to the existing network through firmware upgrades only, unlike other technologies such as massive MIMO that require major hardware upgrades. In cooperation, a user becomes associated with multiple SCs if the achievable data rate by a single SC is not satisfied. However, in the case of Wi-Fi offloading, the UEs satisfy their partial data rate through the Wi-Fi network. Next, we investigate the joint problem of RB and power allocation for cooperation and Wi-Fi offloading strategies.

\subsection{Cooperation in Small-Cell BSs}

In this subsection, we will formulate a problem of sum-rate maximization in cooperative small cellular network and propose a heuristic to solve it by associating a user to multiple SBSs on RBs and by allocating power.

For cooperative SC network, we formulate the problem as a sum-rate maximization (utility maximization) problem. The constraints of QoS, maximum association limit and maximum power of SBS are ensured. The problem is formulated for a time frame $T$ to be solved by a central controller (MBS in this case). It aims to associate multiple SBSs to a user, allocates RBs and assigns power. We use a single variable for SBS association and $\mathrm{RB}$ allocation $x_{i, j}^{k}$ and $P_{i, j}^{k}$ is the power allocation variable. The problem formulated is as follows:

$$
\begin{array}{ll}
\max _{\mathbf{X}, \mathbf{P}} & \sum_{j \in J} \sum_{i \in I} \sum_{k \in K} x_{i j}^{k} R_{i j}^{k} \\
\text { subject to } & \sum_{i \in I} \sum_{k \in K} x_{i j}^{k} R_{i j}^{k} \geq R_{j}^{t h} \quad \forall j \in J, \\
& \sum_{i \in I} x_{i j}^{k} \leq M_{j} \quad \forall j \in J, \forall k \in K \\
& \sum_{j \in J} \sum_{k \in K} x_{i j}^{k} \leq N_{i} \quad \forall i \in I, \\
& \sum_{j \in J} x_{i j}^{k} \leq 1 \quad \forall k \in K, \forall i \in I, \\
& \sum_{j \in J} \sum_{k \in K} x_{i j}^{k} P_{i j}^{k} \leq P_{\max } \quad \forall i \in I .
\end{array}
$$

As already discussed, our objective function as given in (13) is to maximize achievable data rate over all SC UEs. Here multiple SBSs serve a UE. Constraint (14a) is the QoS constraint with $R_{j}^{\text {th }}$ as the minimum data rate requirement of each user $j$. It ensures that the achievable data rate of each user is above a threshold $R_{j}^{\text {th }}$. Constraint (14b) is the cooperative SBS constraint. This constraint dictates the number of SBS that all UEs can be associated with and is less than equal to $M_{j}$. In case the value of $M_{j}$ is 1 , cooperative communication turns into a non-cooperative scenario i.e., each user can only associate itself to a single SBS. Number of UEs that can be served by each SBS is ensured by constraint (14c). Similarly, constraint (14d) ensures that a RB cannot be reused by a SBS. Lastly, constraint (14e) ensures that the maximum power constraint of each SBS is not violated.

Our model of cooperative communication is a mixed integer non-linear programming (MINLP). As illustrated in (13), our objective function is joint resource allocation problem with controlling variables $P_{i j}^{k}$ and $x_{i j}^{k}$. $P_{i j}^{k}$ is a continuous variable whereas $x_{i j}^{k}$ is a binary variable. Our objective function is a non-convex function jointly as well as individually with respect to each variable [33] and hence it is an NP hard problem and is hard to solve 
in polynomial time. Owing to the complexity of the problem, we propose a heuristic algorithm to solve the joint problem in polynomial time.

Sequential Cooperative Rate Enhancement (SCRE) Algorithm for Small-Cell Network

We propose a SCRE algorithm to solve the joint problem of SBS association, RB allocation and power allocation. SCRE is a centralized algorithm and is run on frame-by-frame basis. The MBS solves the problem for a time frame $T$ at the start of the time frame. It assumes to be aware of the quasi-static channel state information for each SBS $i$ to UE $j$ and central node is aware of all the thresholds applied in the network.

The problem is a joint problem of RB allocation, user-to-multiple SBS association and power allocation. These three allocation variables are solved in sequence to maximize the sum rate of the network. Preference is given to the resources of the first SBS that a user is associated with. In case the user's data rate is not satisfied, the association of the edge UEs (decided based on the channel state conditions) increases whereas the power of the near users is decreased. We assume that the channel state information is known at the MBS [34].

Algorithm 1 shows the steps of the centralized SCRE algorithm. First, all the UEs present in the SC network find a single SBS with which it has the best channel gain from distance priority vector $F_{j}$. Without repetition of RBs, UEs associate with their first-priority SBSs as given in line 4 without violating the maximum association of each SC as stated in constraint (14c). After subtraction a certain portion (depending upon how much data rate of user $j$ needs to be enhanced) of the total maximum power, it distributes the power uniformly between all the UEs associated with it. It saves a certain portion to allocate it to newly associated UEs. Now based on the association and power, it computes the achievable data rate of each user as given in (3). The MBS then verifies if constraint (14a) (QoS constraint) is satisfied or not for each user in the network. In case the achievable data rate of a user $j$ is below the rate threshold values $R_{j}^{\text {th }}$, to satisfy constraint (14a) then MBS provides it with more resources i.e., RBs, SBSs or power, as mentioned in line 9. All the resources should be completely used by first best link of UEs. If RBs of SBS $i^{\prime}$ are not used then MBS provides its non-allocated RBs to UE $j$. The second priority SBS $i^{\prime \prime}$ from set $I^{\prime}$ of user $j$ is determined by the central node, where $I^{\prime}$ is a set of $\left\{I-i^{\prime}\right\}$ SBSs and checks if its channel gain difference with first-priority SBS $i^{\prime}$ is less than a threshold value, after this the UE is associated with this SBS.

Our decision of the next resource to be used depends on the difference of channel state of SBS a user is associated with and the next best potential SBS it can be associated with. We use this approach to distinguish most of the edge UEs in the network. In the case the channel gain difference is greater than threshold that means the user is far from the second best SBS and it should not be associated with it. The last resort to satisfy a user's data rate is to provide more power from already associated SBS $i^{\prime}$. After all the options are considered the central node updates the RBs, power and association, and ensures the constraints are satisfied for each user in a round robin fashion. Thus, the resources (RBs, association and power) increase in a sequence based on the channel state conditions to maximize the sum data rate of the network. If UE achieves its data rate then its allocated power is decreased unless its QoS constraint is not violated, and this power adds to available power $\Delta_{i}$ of SBS $i$ it is associated with.

The algorithm SCRE performs for all the UEs in a cooperative SC network. The computational complexity is of the order $O\left((M \times N)\left(M_{j}+2 K\right)+\left(M^{2} \times N\right)(3 K)\right)$.

\subsection{Offloading in Co-Located Wi-Fi-Small-Cell BS}

In this subsection, we will formulate a problem of sum-rate maximization by using Wi-Fi offloading in small cellular network and propose a heuristic to solve it by associating a user to SBSs on LTE, offload it to unlicensed band to meet its data rate demand and allocating power. 


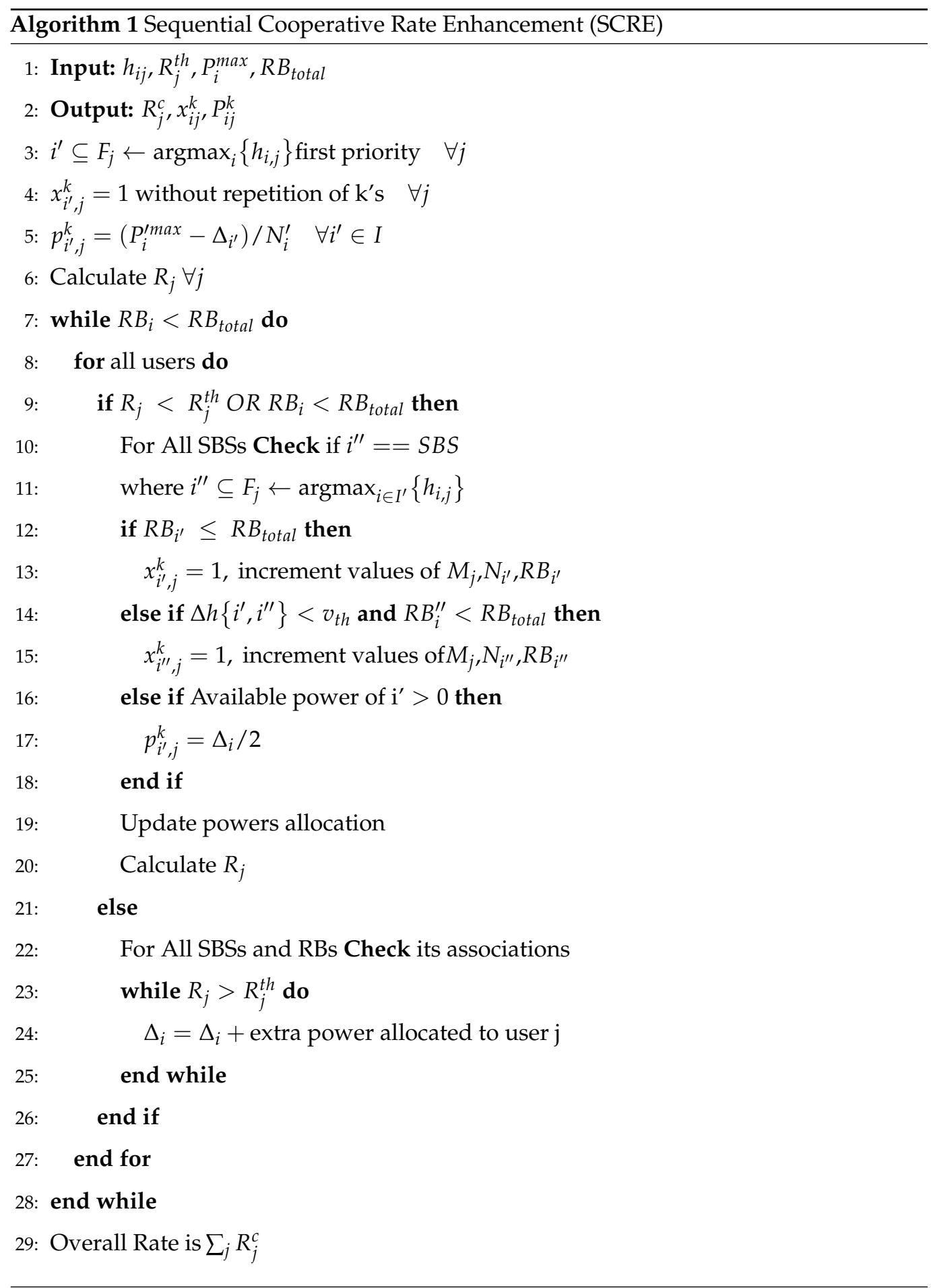

In co-located Wi-Fi-SC network, we formulate the problem as a sum-rate maximization problem as done for cooperative SC network. In this case, the constraints of QoS, maximum Wi-Fi leftover capacity, maximum association limit and maximum power are ensured. For a fair analysis of cooperation and Wi-Fi offloading, this problem is formulated for a time frame $T$ to be solved by a central controller. The central controller directs to associate UE to a single SBS, allocate RBs, offload on Wi-Fi for partial satisfaction of data rate of a user and assigns power. We use single variable for SBS LTE association and RB allocation $y_{i j^{\prime}}^{k}$ single variable for SBS LTE-U association $z_{i j}, R_{i j}^{U}$ for leftover data rate allocation and $P_{i, j}^{k}$ is the power allocation variable. The problem is formulated as: 


$$
\begin{array}{cl}
\max _{\mathbf{Y}, \mathbf{Z}, \mathbf{P}, R_{j}^{U}} & \sum_{j \in J}\left(\sum_{i \in I} \sum_{k \in K} y_{i j}^{k} R_{i j}^{L T E, k}+\sum_{i \in I_{j}} z_{i j} R_{i j}^{U}\right), \\
\text { subject to } & \sum_{j \in J} z_{i j} R_{i j}^{U} \leq\left(R_{A P_{i}}-\sum_{w \in W} r_{i}^{w}\right) \quad \forall i \in I_{j}, \\
& R_{j}^{L T E}+R_{j}^{U} \geq R_{j}^{t h} \quad \forall j \in J, \\
& \sum_{i \in I} y_{i j}^{k} \leq 1 \quad \forall j \in J, k \in K, \\
& \sum_{i \in I_{j}} z_{i j} \leq 1 \quad \forall j \in J, \\
& \sum_{j \in J} \sum_{k \in K} x_{i j}^{k} P_{i j}^{k} \leq P_{\max } \quad \forall i \in I .
\end{array}
$$

As already discussed, our objective function as given in (15) is to maximize achievable rate over all SC UEs. Here a UE is served using additional Wi-Fi capacity from a SC on LTE$\mathrm{U}$ band, therefore, data rate maximization is achieved over both licensed and unlicensed band. Constraint (16a) is the Wi-Fi rate assignment constraint [32]. It ensures that sum achievable data rate of all the cellular UEs on LTE-U band should be less than total leftover capacity $L_{i}$ of the Wi-Fi AP which is co-located with the SBS. All the UEs are candidates to offload which have SBS $i$ in their specific Wi-Fi range and these selected SBSs are in set $I_{j}$ for each of the $j^{\text {th }}$ UE. Constraint (16b) is the QoS constraint with $R_{j}^{t h}$ as the minimum data rate requirement of each UE $j$. It ensures that the achievable data rate of each UE over both LTE and LTE-U links is above a threshold $R_{j}^{\text {th }}$. Constraint (16c) is the user association on LTE and it ensures that a UE can be offloaded onto single SBS. Constraint (16d) is for user association on LTE-U. It also dictates that a UE can be at most associated with single Wi-Fi AP if it is present in its transmission range. Lastly, Constraint (16e) ensures that the maximum power constraint of each SBS is not violated.

Similar to cooperative scenario, Wi-Fi offloading is also a MINLP and is hard to solve in polynomial time. As illustrated in (15), our objective function is a joint resource allocation problem with controlling variables $y_{i j}^{k}, z_{i j}, P_{i j}^{k}$ and $R_{i j}^{U}$. $R_{i j}^{U}$ is continuous variable, it can take any value in between 0 and maximum leftover capacity, $P_{i j}^{k}$ is also continuous variable and it can take any value between 0 and $P_{\max }$ whereas $x_{i j}^{k}$ and $z_{i j}$ are the binary variables. Constraints are also non-convex along with the objective function [33]. As this problem is also NP hard, we propose a heuristic to solve this problem also.

Sequential Offloading Rate Enhancement (SORE) Algorithm for Small-Cell Network

A SORE algorithm is proposed to solve the joint problem of SBS association and RB allocation, power allocation and leftover Wi-Fi rate allocation. Similar to SCRE, SORE is also centralized algorithm and is assumed to be aware of the channel state information for the each SBS $i$ to UE $j$.

The problem is a joint problem of RB allocation, user-SBS association, power allocation and leftover Wi-Fi rate allocation. These four allocation variables are combined and then solved in a sequence to maximize the sum rate of the network. In this case, the preference is given to the resources of the SBS that a user becomes associated with on LTE band. In case the user's data rate is not satisfied, then Wi-Fi offloading is performed to satisfy the partial data rate requirement of the user (decision based on the presence of UE within Wi-Fi range).

Algorithm 2 shows the steps of the centralized SORE algorithm. First, all the UEs are associate to a single SBS with which it has the best channel gain similar to SCRE and then RBs are assigned as represented in line 4 . A significant proportion of the total power of each SBS $\bar{i}$ is then divided uniformly between associated UEs in line 5. The data rate requirement of each user is calculated and verified according to (8). Secondly, distance 
priority matrix $I_{j}$ is made for all the UEs in which SBSs with minimum distance with user $j$ are placed in ascending order as illustrated in line 7. Data rate of UEs in the network is required to be satisfied according to constraint (16b). If the value is below than rate threshold value, then it needs to provide more resources (RBs, Wi-Fi Offloading or more power) as mentioned in line 10. First, it lies on the first-priority SBS $\bar{i}$ for more resources. All the licensed resources must be exhausted before Wi-Fi offloading is done. Next it checks SBS for UE $j$ from distance priority matrix, if UE lies within Wi-Fi range of that SBS and this SBS also has non-zero leftover capacity then it provides percentage of leftover rate depending on data rate requirement of UE $j$. The remaining leftover capacity is reserved for other UEs that lie within its Wi-Fi range. One last way to enhance data rate is to provide more power from already associated SBS $\bar{i}$. After meeting all these requirements, the user data rate is adjusted and the central node is required to allocate power to the new RBs from the available power of the associated SBS, and it then again calculates the rate iteratively according to (11). Lastly, it will update number of RBs used, leftover capacity and the available power to each SBS. Each UE satisfies its QoS until all the resources are used. The SORE algorithm is applied to all the UEs in the co-located Wi-Fi-SC network. The order of complexity of SORE is $O\left((M \times N)(2 K)+\left(M^{2} \times N\right)(3 K)\right)$.

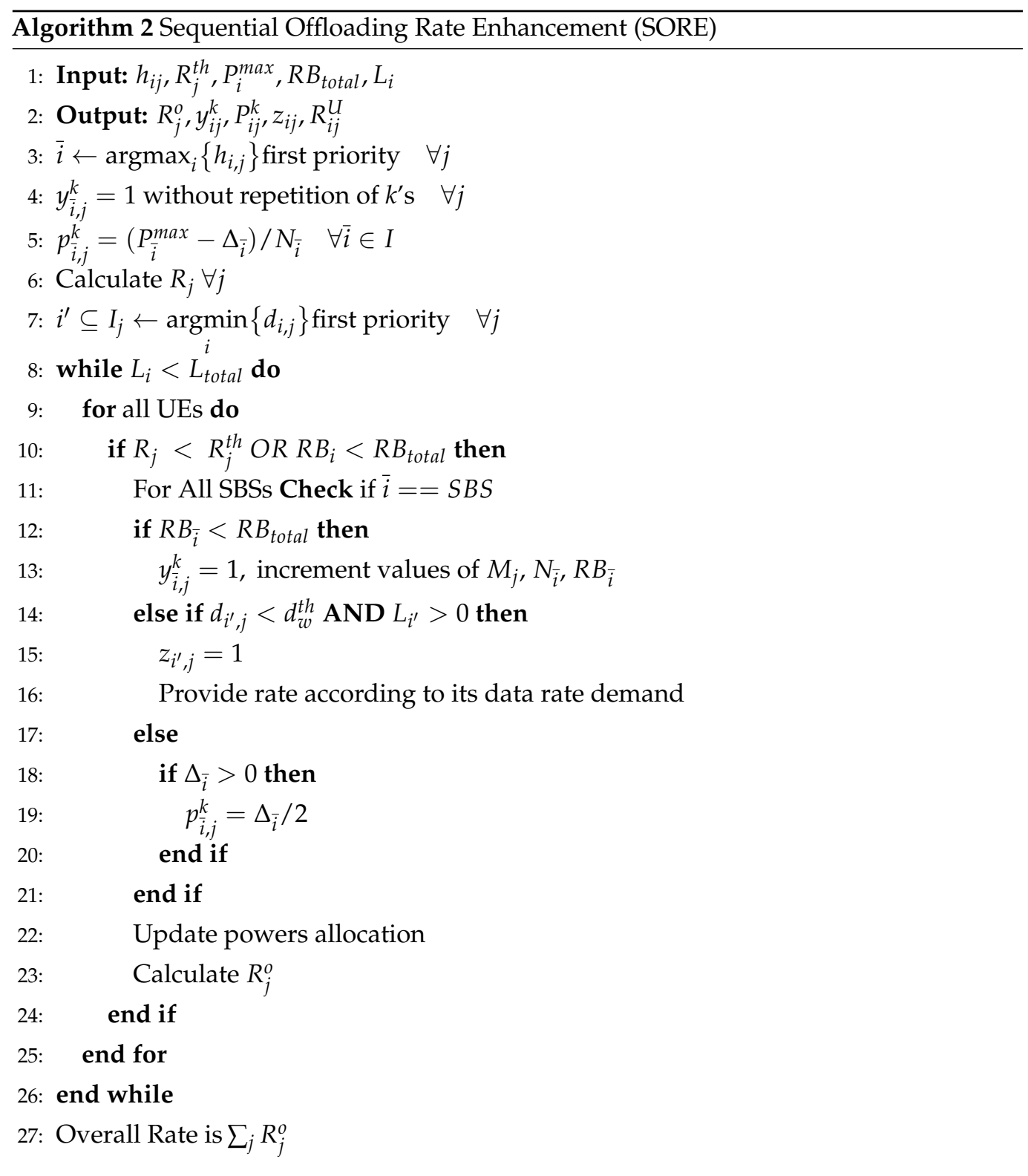




\subsection{Switching between SCRE and SORE}

Figure 2 shows the switching strategy employed by the network. The network performs switching between Wi-Fi offloading and cooperative communication depending on the network conditions. In this network the data rate of each user can be enhanced by either serving it with more than one SC base stations simultaneously, i.e., cooperative communication or by offloading the traffic to the unlicensed band which is also used by the Wi-Fi. In this network, switching mainly depends on the data rate requirement and user's density. As shown in Figure 2, SCs bounded with dashed lines are present in a MC.

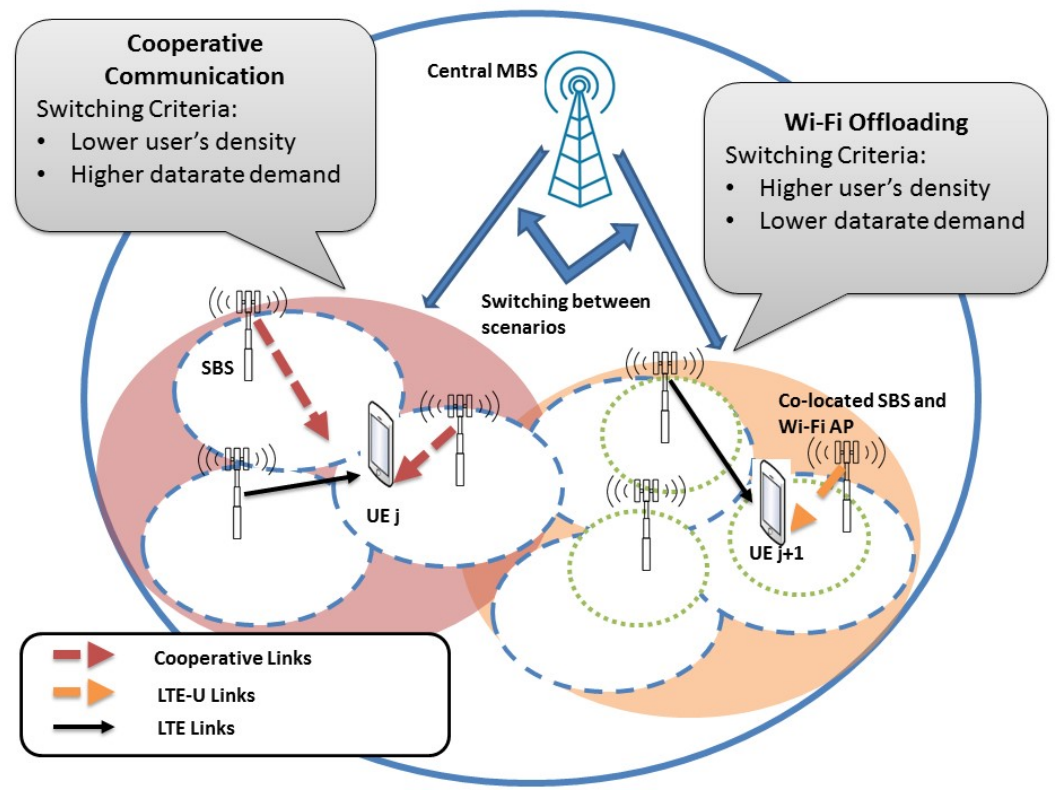

Figure 2. Switching Model Between Cooperation Communication and Wi-Fi Offloading.

Based on data rate demand of users and user's density, central BS (Macro BS) switches to the scheme that performs better under different conditions. As shown in figure, UE $j$ is served cooperatively by connecting to its own SBS using LTE link and then additionally connecting to two neighboring SBS using cooperative communication. Similarly, UE $j+1$ is served using unlicensed Wi-Fi band in addition to its own LTE link. Next we present a switching algorithm that tries to maximize the user data rates while switching between the SCRE and SORE while considering the network conditions.

Switching between Cooperation and Offloading (SCO) Algorithm

Similar to SCRE and SORE, SCO is also a centralized algorithm and is assumed to be aware of the channel state information between each BS $i$ to UE $j$, switching criteria, user density and their data rate demand. Algorithm 3 shows the switching logic of SCO. The inputs to this algorithm are rate thresholds $R_{j}^{t h}$, maximum power $P_{i}^{\max }$ of each SBS $i$, total number of RBs $R B_{\text {total }}$, leftover rate $L_{i}$ of each SBS $i$, total number of UEs $N$, switching parameter for data rate threshold $\alpha$ and switching parameter for user density $\beta$. Similar to SCRE and SORE, in the SCO algorithm the user is associated with the SBS with the highest channel gain. Then its data rate is calculated according to (3) or (8). If the data rate requirement is not fulfilled through connection with a single SBS and more resources need to be allocated, then the SCO algorithm switches to either SCRE or SORE to fulfill the additional data rate requirement. 


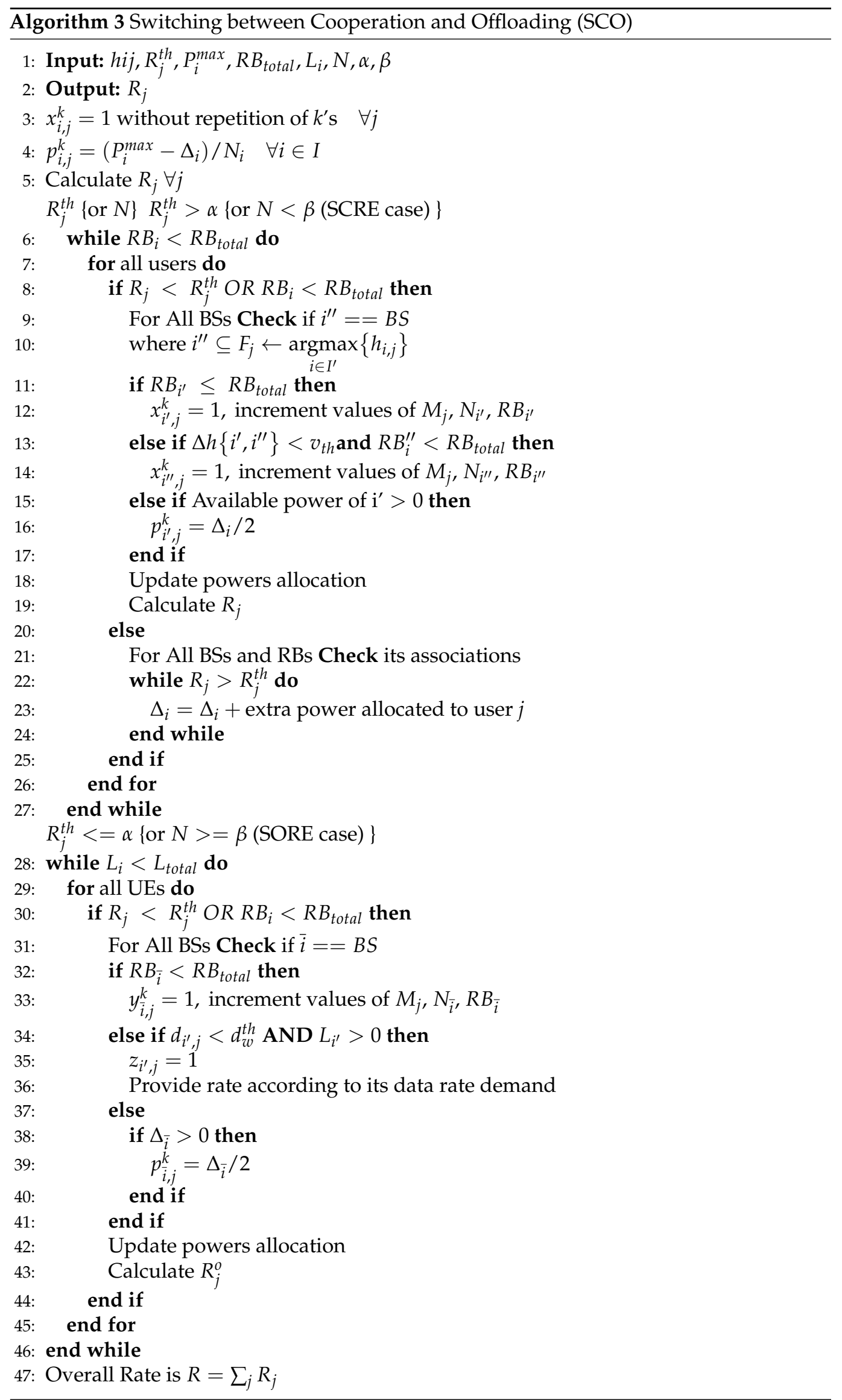


We consider two network parameters before switching, i.e., user data rate requirement and user density. The data rate threshold and user density is used to perform switching to enhance the data rate in the small-cell network. After executing SCRE and SORE algorithms, the data rate threshold $\alpha$ and user density $\beta$ are computed, which are then used by SCO algorithm to switch to either SCRE or SORE ( $\alpha$ and $\beta$ are called switching parameters). Depending upon the switching parameters, when SCO algorithm switches to SCRE, it executes the main while loop for SCRE (from line 7 to 28). Similarly, when SCO switches to SORE, it executes the main while loop for SORE (from line 8 to 26). After the switching operation, we obtain the achievable data rate $R_{j}$ for each user $j$. Lastly, the achievable data rate of the network is calculated.

The SCO algorithm is applied to all the UEs in SC network. The order of complexity of SCO is $O\left(5(M \times N)+2(M \times N \times K)\left(1+4 M_{j}+K(3(M \times N)+8)\right)\right.$.

\section{Performance Evaluation}

In this section, we perform a comparative analysis between SCRE and SORE algorithms proposed in Section 4. First, these proposed algorithms are analyzed with respect to the most important factors, i.e., maximum association limit in case of SCRE and Wi-Fi leftover capacity in case of SORE. Then comparison is performed between SCRE and SORE by varying different network parameters.

Comparison: To the best of our knowledge, no other work has performed this comparison under these conditions, therefore, it is difficult to compare our results with any prior state of the art. Instead, we compared the two proposed schemes with a baseline case of no offloading and no cooperation. We performed this comparison to observe the increase in gain (in terms of average data rate) that each of these schemes offer with reference to the baseline.

We consider a small portion of macro cell with small cells with approximate radii of $70 \mathrm{~m}$. A total of 15 small cells are uniformly distributed. All the presented results are computed with a 95\% confidence interval by averaging over $10^{3}$ different channel conditions. To simplify our computations, we calculate normalized data rates. Table 2 summarizes the values of the parameters used for evaluation. Different values of the network parameters have been used in simulations, as shown in Table 2, to represent variation in network conditions.

Figure 3 demonstrates the effect of increase in cooperation (number of small cells a user can associate itself with) on the achievable data rate per user and the consumed power per associated SBS per user. We consider 15 SBSs, 7 RBs and 5 users. We observe that with the increase in the associated SBSs, the achievable data rate per user increases. This increase is significant in the start (from $M_{j}=1$ to $M_{j}=2$ ) and gradually flattens. This indicates that after $M_{j}=3$, there is not many resources (to increase achievable data rate) left in the system to be exploited by cooperation in this scenario. For practical scenario, its maximum value can be 3 or 4 as most of the SCs are surrounded by these many neighboring SCs. We also observe that the consumed power of each SBS per user decreases with the increase in association of the users. This decrease is as a result of being served by multiple SBS which distributes the power consumption between different SBSs. Please note that the algorithm is not violating the maximum power constraint of each SBS. Thus, cooperation increases the achievable data rate per user and decreases the power consumed per SBS per user.

Figure 4 presents the performance analysis of SORE algorithm for Wi-Fi offloading. Here users are offloaded to Wi-Fi network if they are unable to fulfill their data rate demand by connecting with single cellular BS. We consider 5 SBSs, 5 RBs and 20 users. We analyze the effect of changing the total leftover capacity of each Wi-Fi AP on the achievable data rates and transmission power of cellular network. It is pertinent to note that the improvement in performance reflects the amount of leftover rate of each $\mathrm{Wi}-\mathrm{Fi}$ AP. If this amount is high enough then the users obtain higher partial data rates when offloaded to Wi-Fi network. We analyze achievable data rates of network at two different 
rate threshold values by increasing leftover capacities of each Wi-Fi AP present in the Wi-Fi network. The results indicate that by increasing the leftover capacity, the cellular UEs obtain better data rates. When the UEs data rate requirement is less, i.e., $R^{t h}$ equals to 50 , then they are satisfied by the cellular resources only, as shown by the red dashed line. On the other hand, the consumed cellular power per SBS per user is not affected much with the increase in the Wi-Fi leftover capacity. Hence, the performance of SORE is mainly driven by the Wi-Fi leftover capacity; however, the power consumption per SBS per user is hardly affected.

Table 2. Evaluation Parameters.

\begin{tabular}{ccc}
\hline Parameters & Symbols & Values \\
\hline Maximum power & $P_{\max }$ & $23 \mathrm{dBm}$ \\
No. of SBSs & $M$ & $3-15$ \\
No. of UEs & $N$ & $5-30$ \\
No. of RBs & $K$ & $5-30$ \\
Rate threshold & $R^{t h}$ & $50-300 \mathrm{bits} / \mathrm{s}$ \\
Noise variance & $\sigma^{2}$ & $1.0 \times 10^{-15}$ \\
Saved power of SBS i & $\Delta_{i}$ & 0.025 Watts \\
Min channel difference threshold & $v^{\text {th }}$ & 0.5 \\
\hline
\end{tabular}

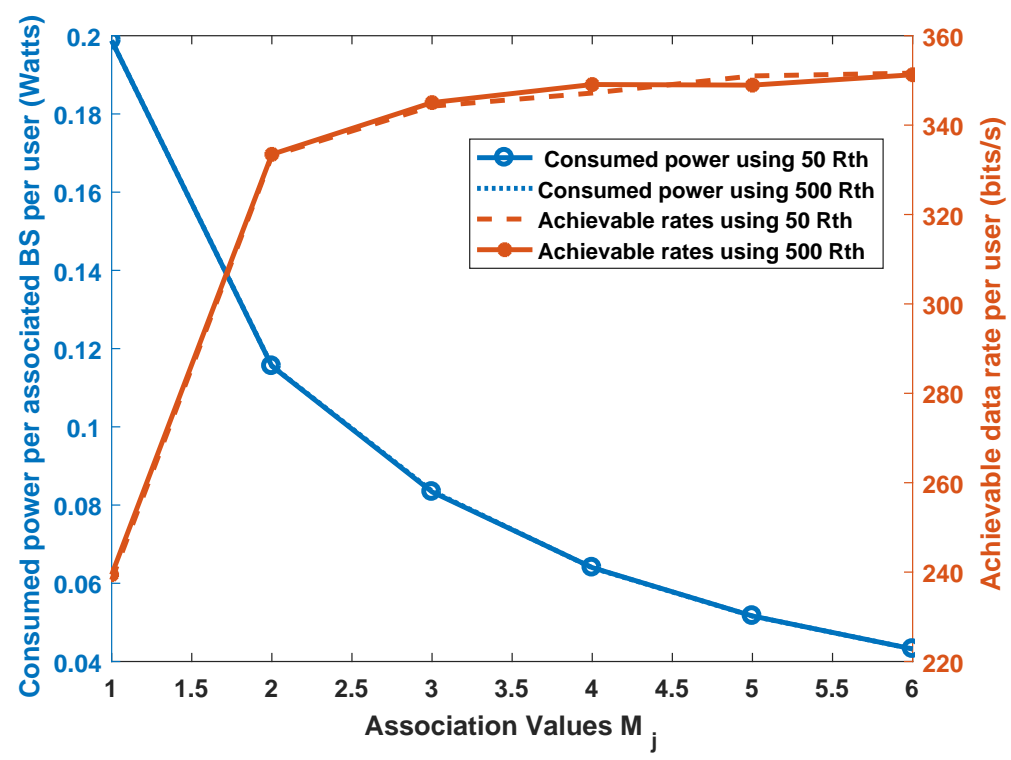

Figure 3. Analysis of Rate allocation in Cooperation regarding Association.

Next, the simulation results in Figure 5 shows the effect of the increase in user density on the achievable data rate per user of SCRE and SORE in the SC network. We evaluate these results with 3 SBSs, 10 RBs and 100 bits/s rate threshold value. As the number of UEs increases from 5 to 30, a significant decrease in the achievable date of both SCRE and SORE is observed. This decrease in the achievable data rate is due to increase in the number of UEs without changing the resources present in the network, therefore reducing the resources per UE. For SCRE which is represented by a dashed magenta line in Figure 5, the QoS per user degrades and is similar to non-cooperative/Wi-Fi offloading scenario. However, as the density of the user increases, the QoS per user of cooperation achieves the QoS per user in non-cooperative/Wi-Fi offloading case. This is due to the of the maximum number of SBS to user association constraint. As the number of UEs increase, a UE cannot be associated with other SBS because each SBS can barely serve its own users, and there is no space left for cooperation. Similar to SCRE, the performance of SORE is significantly better than non-cooperative/Wi-Fi offloading case in a less dense network, whereas in a dense network the increase in QoS per user as compared to non-cooperative/Wi-Fi 
offloading case depends on the Wi-Fi leftover rate. In other words, if the Wi-Fi network is crowded, the leftover capacity that it can share with LTE UEs is less and thus the increase in data rate is less compared to a less crowded Wi-Fi network. The performance of SCRE is better as compared to SORE in a less dense environment. However, with the increase in the number of UEs, the performance of SCRE becomes worse than the performance of SORE. The performance degradation in SCRE as compared to SORE is dependent on the density of the Wi-Fi network (or the leftover Wi-Fi rate $L_{i}$ ). With higher leftover rates value, SORE performance becomes better. Thus, we can conclude that cooperation outperforms Wi-Fi offloading in a less dense network whereas Wi-Fi offloading is more advantageous in a dense SC network, provided there is sufficient leftover Wi-Fi capacity available. The percentage increase in rate of Wi-Fi offloading is $43 \%$ and of cooperation is $32 \%$ as compared to non-cooperative/Wi-Fi method. After executing both SCRE and SORE, the value of switching parameter for user density $\beta$ is passed to SCO to perform switching. The performance of SCO is shown as a dotted green line which is better than the performance of either of SCRE and SORE. It also shows that for $\beta<=10$ (less dense network), it performs similar to SCRE and for $\beta>10$ (dense network), it performs similar to SORE.

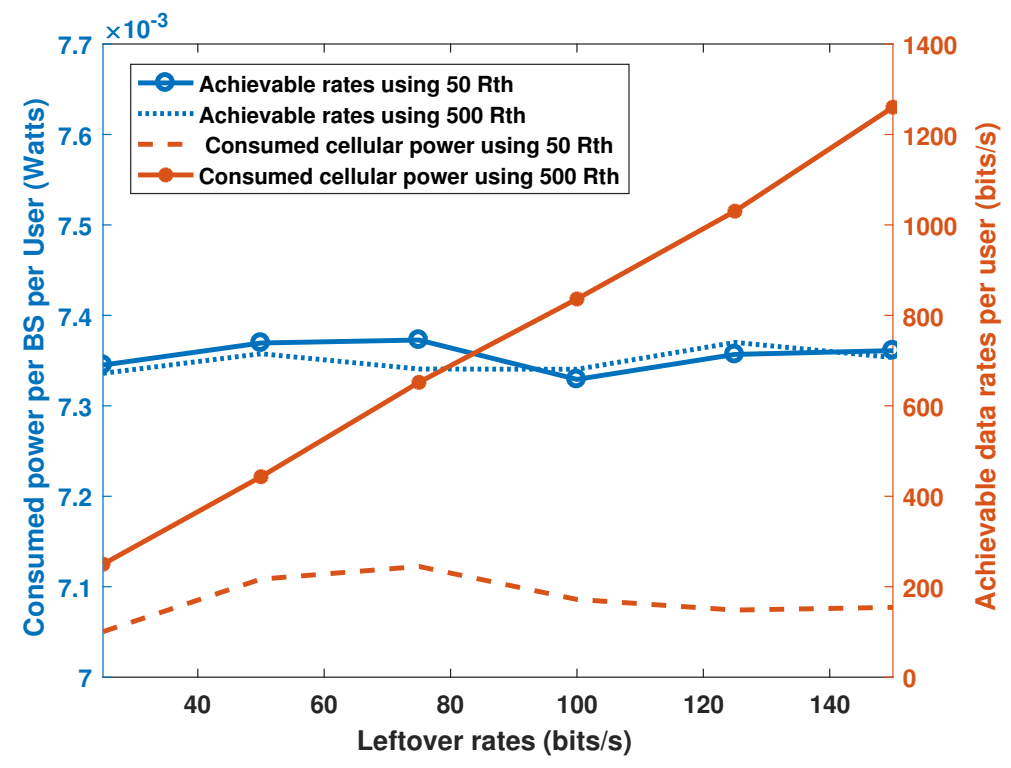

Figure 4. Analysis of rate allocation in Wi-Fi Offloading regarding leftover rate.

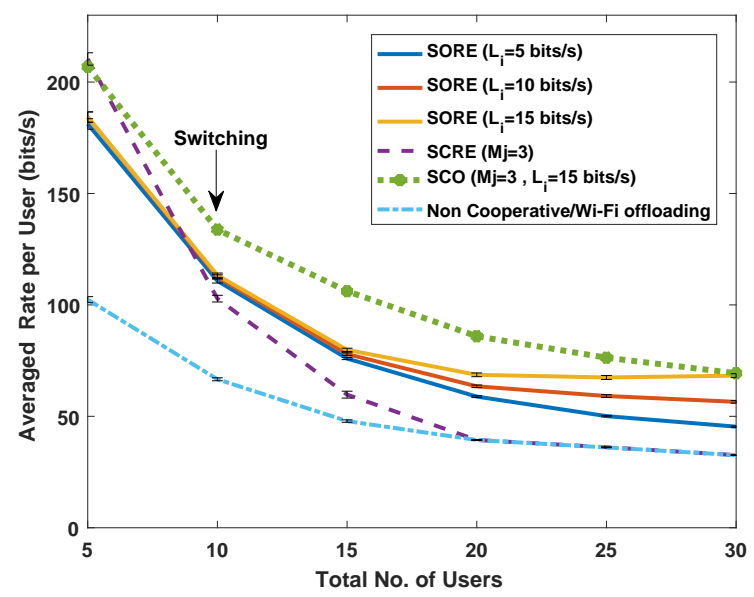

Figure 5. Averaged Achievable data rates per user by changing No. of Total UEs in Cooperative Communication, Without Cooperative Communication and by taking 5, 10, 15 leftover capacity in Wi-Fi offloading and overall 3 SBSs. 
Figure 6 shows the effect of increase in rate threshold on the QoS per user of SCRE and SORE. These results are evaluated using 10 UEs, 3 SBSs and 10 RBs. With the increase in rate threshold, the performance of SCRE increases significantly when the number of UEs in the network is less $(N=5,10)$, whereas only a slight increase is observed for a greater number of users $(N=20)$. However, the performance SORE is nearly constant with increase in the rate threshold. This near constant behavior indicates that the performance of SORE mainly depends upon the leftover Wi-Fi rate and not the rate threshold of UEs. SCRE outperforms SORE for higher QoS requirement, as associating with multiple SBS helps in using their resources (spectrum, power), which is more beneficial than using the leftover capacity of a single Wi-Fi AP. Thus, we can conclude that the performance of Wi-Fi offloading is mainly influenced by the Wi-Fi leftover capacity, whereas cooperation is more beneficial when the data rate requirement is higher in a less dense network. After executing both SCRE and SORE, the value of switching parameter for rate threshold $\alpha$ is passed to SCO that performs switching. The green dotted line in Figure 6 indicates that for $\alpha<=100$ (lower data rate demand), the performance of SORE is better and for $\alpha>100$ (higher data rate demand), the performance of SCRE is better. Hence, SCO switches to the better rate enhancement technique.

Figure 7 shows a relationship between average data rate per UE and total number of RBs per SBS used in network. We analyze cooperation while varying the number of UEs i.e., $N=10,20$ and 30 . For Wi-Fi offloading we consider $N=20$. The rate threshold is 100 bits / s and 3 SBS are considered. An increasing trend is observed with the increase in number of RBs in both SCRE and SORE. Highest increase in data rate is observed for SCRE with $N=10$ users. On the other hand, SORE still shows a significant increase with RBs.

These results are for 'snapshot-based' static load conditions and may not be representative of the real situation. However, this quantification of results for various network parameters provides useful insight on set of conditions for network offloading. As we have simulated both high density as well as low density networks under different traffic requirements, the general conclusions drawn from this study are still valid when we have dynamic traffic. It is true that offloading decisions cannot be based on per slot basis because it requires a lot of overhead, and a long-term approach is useful to make such decisions; however, the conditions to select offloading method remain the same.

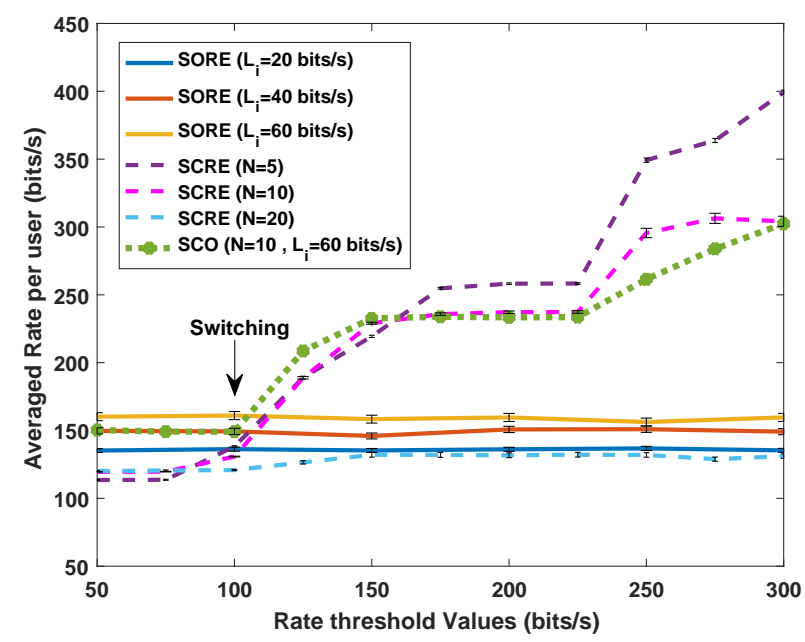

Figure 6. Averaged Achievable data rates per user by changing Rate threshold values in Cooperative Communication and by taking 20, 40, 60 leftover capacity in Wi-Fi offloading and overall 3 SBSs, 10 UEs and 10 RBS. 


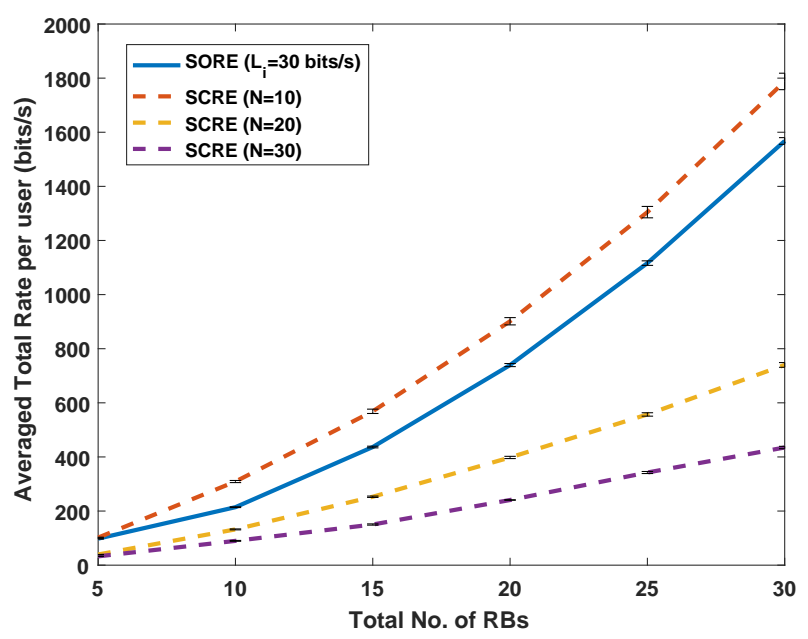

Figure 7. Averaged Achievable data rates per user by changing No. of Total RBs using 10, 20, 30 UEs in Cooperative Communication and 20 UEs with 30 leftover capacity in Wi-Fi offloading and overall 3 SBSs.

\section{Conclusions}

Unlicensed accessed is one of the key enablers for $5 \mathrm{G}$ technologies as service requirements on licensed bands are becoming stringent and licensed bands alone ae unable to meet these demands. Co-existence studies for unlicensed and licensed bands are part of 3GPP Release 15 and other future releases that are trying to exploit unlicensed bands whenever infrastructure allows it. In this paper, we have presented a comparison between two rate enhancement strategies, i.e., SBS cooperation and Wi-Fi Offloading based on network parameters, such as user density and rate threshold. For this, we consider maximum rate joint optimization problem for user association, resource block allocation and power allocation for cellular UEs in cooperative communication scenario. However, for Wi-Fi offloading scenario, resource block and power allocation is considered along with the Wi-Fi leftover capacity. The results indicate when UEs are unable to satisfy their rate constraint, they can enhance their data rate using either of the two heuristics depending on the network condition. SBS cooperation (SCRE) is more beneficial in a less dense network where several UEs are likely to be present at the cell edge and when the average user data rate requirement is higher. Wi-Fi offloading (SORE) is more advantageous when the density of cellular UEs is higher and significant number of UEs are present in the Wi-Fi coverage. Moreover, an SCO algorithm is also proposed that optimally switches between SCRE and SORE depending on network conditions. Our results quantify the set of conditions when Wi-Fi unlicensed band and (licensed) intercell cooperation could be used to meet tough rate requirements from the data hungry services, such as video streaming.

Author Contributions: Conceptualization, S.J. and F.A.B.; Formal analysis, A.A., S.J. and M.M.B.; Funding acquisition, F.A.B.; Investigation, S.J.; Methodology, A.A.; Project administration, F.A.B.; Writing-original draft, A.A., S.J. and F.A.B.; Writing-review and editing, M.M.B. and A.R.M. All authors have read and agreed to the published version of the manuscript.

Funding: This work has emanated from the research funded by Higher Education Commission (HEC), Pakistan, under grant No. HEC NRPU 20-4339/RED/HEC/14/235.

Acknowledgments: The authors express deep gratitude to Faizan Ahmad (Assist. Mathematics Dept, IST) for his valuable guidance and feedback in this research work.

Conflicts of Interest: The authors declare no conflict of interest. 


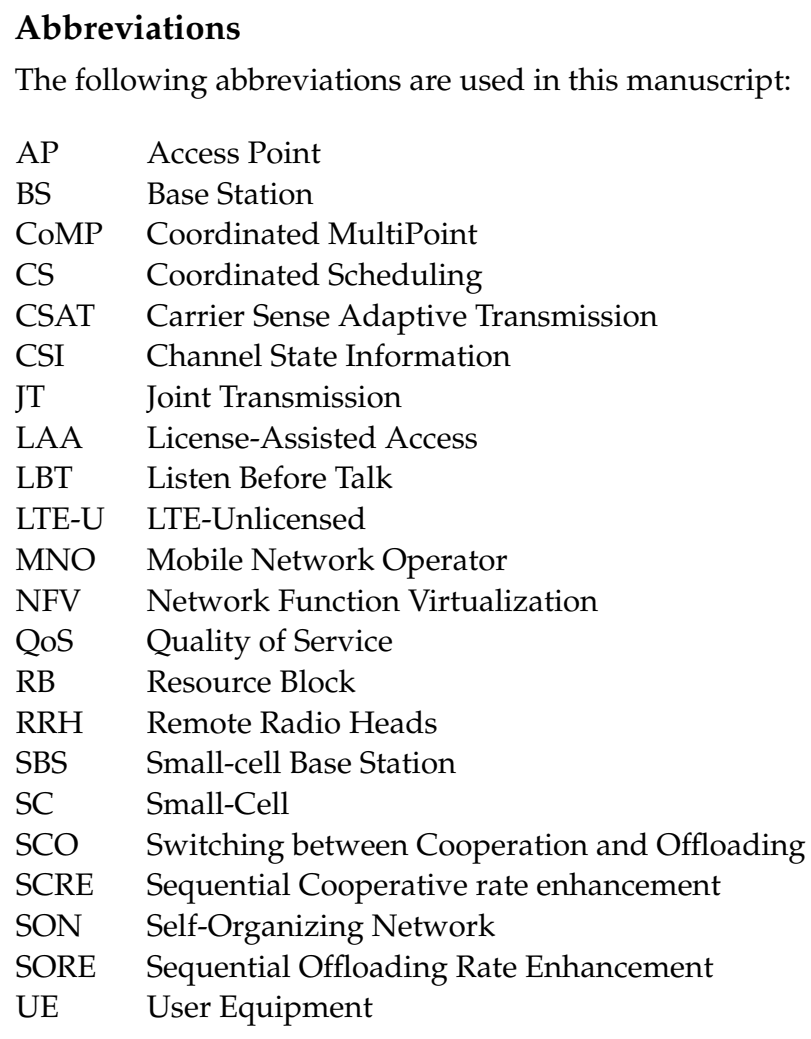

\section{References}

1. Labib, M.; Marojevic, V.; Reed, J.H.; Zaghloul, A.I. Extending LTE into the unlicensed spectrum: Technical analysis of the proposed variants. IEEE Commun. Stand. Mag. 2017, 4, 31-39. [CrossRef]

2. Han, S.; Liang, Y.C.; Chen, Q.; Soong, B.H. Licensed-assisted access for LTE in unlicensed spectrum: A MAC protocol design. IEEE J. Sel. Areas Commun. 2016, 34, 2550-2561. [CrossRef]

3. LTE-U Forum. Available online: http:/ / www.lteuforum.org/ (accessed on 10 December 2019).

4. $\quad$ ETSI. LTE; Evolved Universal Terrestrial Radio Access (E-UTRA); Physical Layer Procedures. ETSI TS. 2017; pp. 136-213. Available online: https:/ /www.etsi.org/deliver/etsi_ts/136300_136399/136300/09.04.00_60/ts_136300v090400p.pdf (accessed on 12 April 2020).

5. Federal Communications Commission. Office of Engineering and Technology and Wireless Telecommunications Bureau Seek Information on Current Trends in LTE-U and LAA Technology; Federal Communications Commission: Washington, DC, USA, 2015.

6. Zinno, S.; Di Stasi, G.; Avallone, S.; Ventre, G. On a fair coexistence of LTE and Wi-Fi in the unlicensed spectrum: A Survey. Comput. Commun. 2018, 115, 35-50. [CrossRef]

7. Qamar, F.; Dimyati, K.B.; Hindia, M.N.; Noordin, K.A.B.; Al-Samman, A.M. A comprehensive review on coordinated multi-point operation for LTE-A. Comput. Netw. 2017, 123, 19-37. [CrossRef]

8. 3GPP Forum. Available online: http:/ / www.3gpp.org/hetnet (accessed on 4 November 2019).

9. Bassoy, S.; Farooq, H.; Imran, M.A.; Imran, A. Coordinated multi-point clustering schemes: A survey. IEEE Commun. Surv. Tutor. 2017, 19, 743-764. [CrossRef]

10. Tao, X.; Xu, X.; Cui, Q. An overview of cooperative communications. IEEE Commun. Mag. 2012, 50, 65-71.

11. Kim, S.; Choi, J.W.; Shim, B. Feedback Reduction for Beyond 5G Cellular Systems. In Proceedings of the IEEE International Conference on Communications (ICC), Shanghai, China, 20-24 May 2019; pp. 1-6.

12. Li, X.; Zhang, X.; Zhou, Y.; Hanzo, L. Optimal Massive-MIMO-Aided Clustered Base-Station Coordination. IEEE Trans. Veh. Technol. 2021, 3, 2699-2712. [CrossRef]

13. Hamdi, R.; Qaraqe, M. Power Allocation and Cooperation in Cell-Free Massive MIMO Systems with Energy Exchange Capabilities. In Proceedings of the IEEE 91st Vehicular Technology Conference (VTC), Antwerp, Belgium, 25-28 May 2020; pp. 1-5.

14. Björnson, E.; Sanguinetti, L. Scalable Cell-Free Massive MIMO Systems. IEEE Trans. Commun. 2020, 7, 4247-4261. [CrossRef]

15. Okuyama, T.; Suyama, S.; Nonaka, N.; Okumura, Y.; Asai, T. Outdoor Experimental Trials of Millimeter-Wave Base Station Cooperation with Digital Beamforming in High-Mobility Environments for 5G Evolution. In Proceedings of the IEEE 92nd Vehicular Technology Conference (VTC), Victoria, BC, Canada, 18 November-16 December 2020; pp. 1-5.

16. Cui, Q.; Ni, W.; Li, S.; Zhao, B.; Liu, R.P.; Zhang, P. Learning-Assisted Clustered Access of 5G/B5G Networks to Unlicensed Spectrum. IEEE Wirel. Commun. 2020, 1, 31-37. [CrossRef]

17. Han, T.; Li, S.; Zhong, Y.; Bai, Z.; Kwak, K. 5G Software-Defined Heterogeneous Networks With Cooperation and Partial Connectivity. IEEE Access 2019, 7, 72577-72590. [CrossRef] 
18. Chen, J.; Ge, X.; Zhong, Y.; Li, Y. A Novel JT-CoMP Scheme in 5G Fractal Small Cell Networks. In Proceedings of the IEEE Wireless Communications and Networking Conference (WCNC), Marrakesh, Morocco, 15-18 April 2019; pp. 1-7.

19. Chen, Y.; Li, J.; Chen, W.; Lin, Z.; Vucetic, B. Joint user association and resource allocation in the downlink of heterogeneous networks. IEEE Trans. Veh. Technol. 2016, 65, 5701-5706. [CrossRef]

20. Jang, J.; Yang, H.J.; Jwa, H. Resource Allocation and Power Control in Cooperative Small Cell Networks in Frequency Selective Channels with Backhaul Constraint. IEEE Trans. Veh. Technol. 2019, 68, 10926-10942. [CrossRef]

21. You, L.; Yuan, D. Load optimization with user association in cooperative and load-coupled LTE networks. IEEE Trans. Wirel. Commun. 2017, 16, 3218-3231. [CrossRef]

22. Park, J.; Kim, K.S. Load-Balancing Scheme With Small-Cell Cooperation for Clustered Heterogeneous Cellular Networks. IEEE Trans. Veh. Technol. 2018, 67, 633-649. [CrossRef]

23. Zhu, Q.; Wang, X.; Qian, Z. Energy-Efficient Small Cell Cooperation in Ultra-Dense Heterogeneous Networks. IEEE Commun. Lett. 2019, 23, 1648-1651. [CrossRef]

24. Ao, W.C.; Psounis, K. Fast Content Delivery via Distributed Caching and Small Cell Cooperation. IEEE Trans. Mob. Comput. 2018, 17, 1048-1061. [CrossRef]

25. Khan, B.S.; Jangsher, S.; Qureshi, H.K.; Mumtaz, S. Energy Efficient Caching in Cooperative Small Cell Network. In Proceedings of the IEEE Annual Consumer Communications \& Networking Conference (CCNC), Las Vegas, NV, USA, 11-14 January 2019.

26. Zhao, Y.; Xu, K.; Zhong, Y.; Li, X.-Y.; Wang, N.; Su, H.; Shen, M.; Li, Z. Incentive mechanisms for mobile data offloading through operator-owned WiFi access points. Comput. Netw. 2020, 174, 107226. [CrossRef]

27. Fan, W.; Han, J.; Yao, L.; Wu, F.; Liu, Y. Latency-energy optimization for joint WiFi and cellular offloading in mobile edge computing networks. Comput. Netw. 2020, 181, 107570. [CrossRef]

28. Shen, H. Congestion-aware WiFi offload algorithm for 5G heterogeneous wireless networks. Comput. Commun. 2020, 164, 69-76.

29. Zhou, H.; Chen, X.; He, S.; Chen, J.; Wu, J. DRAIM: A Novel Delay-Constraint and Reverse Auction-Based Incentive Mechanism for WiFi Offloading. IEEE J. Sel. Areas Commun. 2020, 38, 711-722. [CrossRef]

30. Feng, G.; Xia, F.; Zhang, Y.; Su, D.; Lv, H.; Wang, H.; Lv, H. Optimal Cooperative Wireless Communication for Mobile User Data Offloading. IEEE Access 2018, 6, 16224-16234. [CrossRef]

31. Wildman, J.; Weber, S. On protocol and physical interference models in Poisson wireless networks. IEEE Trans. Wirel. Commun. 2018, 17, 808-821. [CrossRef]

32. Elsherif, A.R.; Chen, W.P.; Ito, A.; Ding, Z. Resource allocation and inter-cell interference management for dual-access small cells. IEEE J. Sel. Areas Commun. 2015, 33, 1082-1096. [CrossRef]

33. Evangelista, J.V.; Sattar, Z.; Kaddoum, G.; Chaaban, A. Fairness and Sum-Rate Maximization via Joint Channel and Power Allocation in Uplink SCMA Networks. IEEE Trans. Wirel. Commun. 2019, 18, 5855-5867. [CrossRef]

34. Gandotra, P.; Jha, R.K. Device-to-device communication in cellular networks: A survey. J. Netw. Comput. Appl. 2016, 17, 99-117. [CrossRef] 